Supplement of Hydrol. Earth Syst. Sci., 24, 2003-2016, 2020

https://doi.org/10.5194/hess-24-2003-2020-supplement

(C) Author(s) 2020. This work is distributed under

the Creative Commons Attribution 4.0 License.

(c) (1)
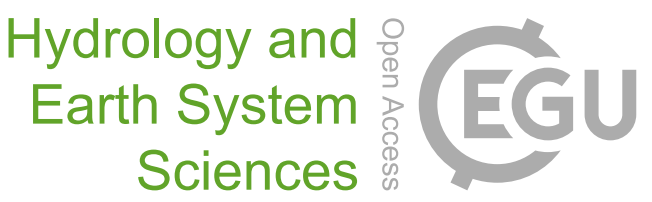

Supplement of

\title{
Revisiting extreme precipitation amounts over southern South America and implications for the Patagonian Icefields
}

\section{Tobias Sauter}

Correspondence to: Tobias Sauter (tobias.sauter@fau.de)

The copyright of individual parts of the supplement might differ from the CC BY 4.0 License. 


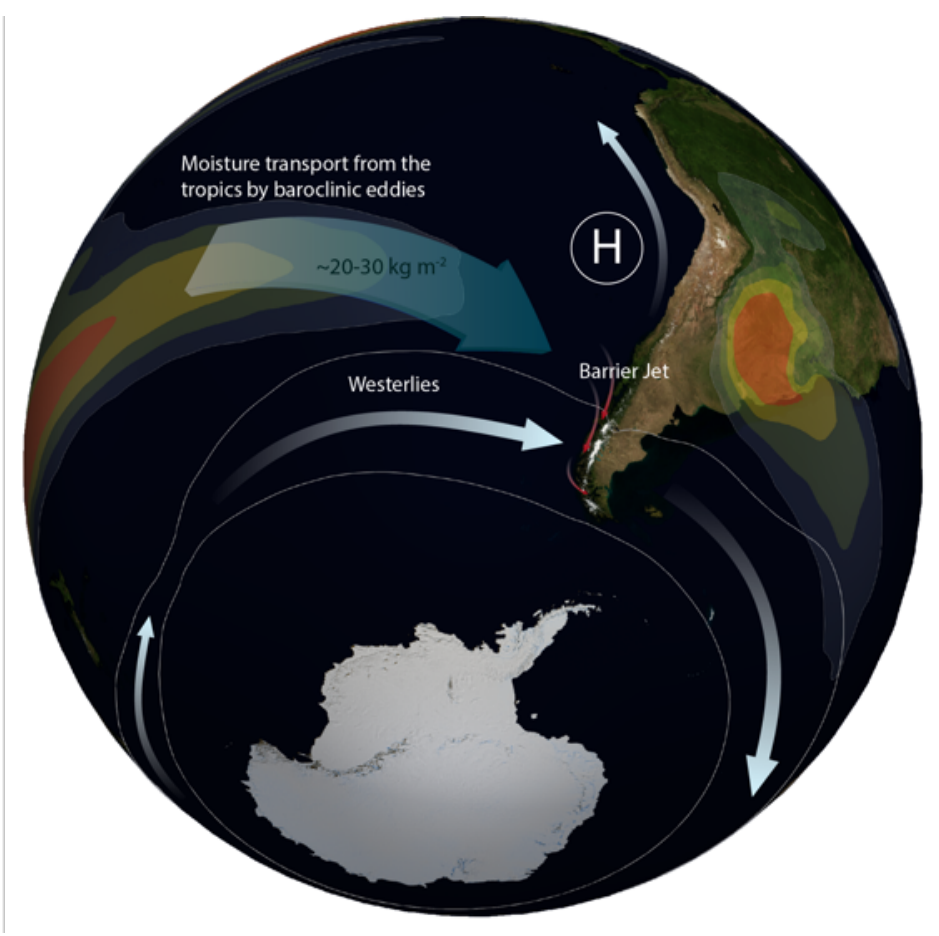

Figure S1: Schematic illustration of the atmospheric large-scale circulation and moisture transport in the South Pacific. Shown are the location of the westerlies for austral summer (December-January-February), the barrier jet along the Andes (red arrows), and the mean moisture transport by baroclinic eddies (blue shaded arrow). The shading indicates regions of high water vapor variability, derived from the 5 ERA interim data for the period 1990-2016 (Dee et al., 2011). The color of the Earth's surface is from NASA's 'Blue Marble' image (CNASA). 


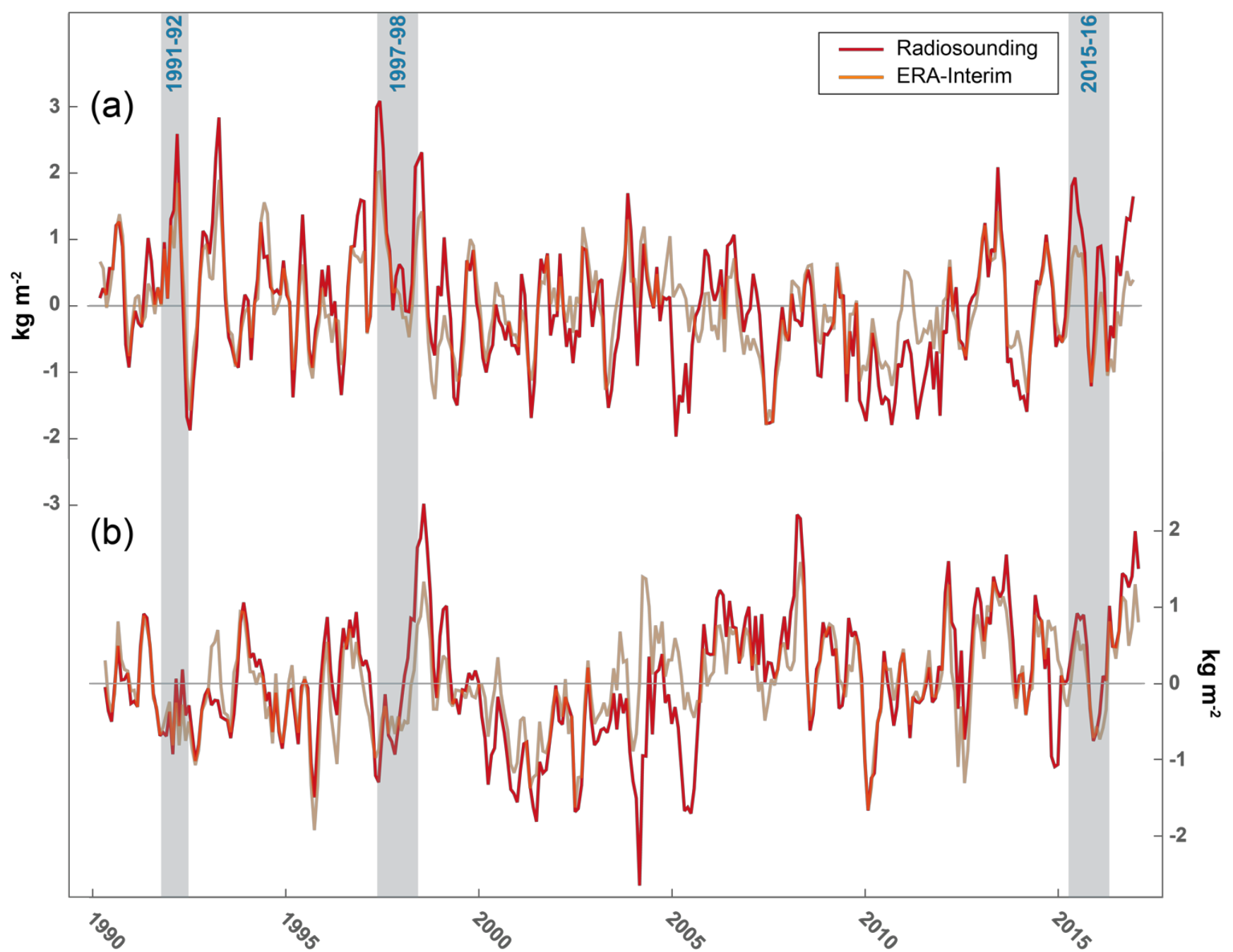

Figure S2: Monthly IWV anomalies in Puerto Montt (a) and Punta Arenas (b). Shown are the running 3-month mean IWV anomalies for the atmospheric soundings and the nearest ERA-Interim grid point from 1990-2016. The blue shaded areas indicate very strong El Niño events $(\mathrm{ONI}>1.5)$. 


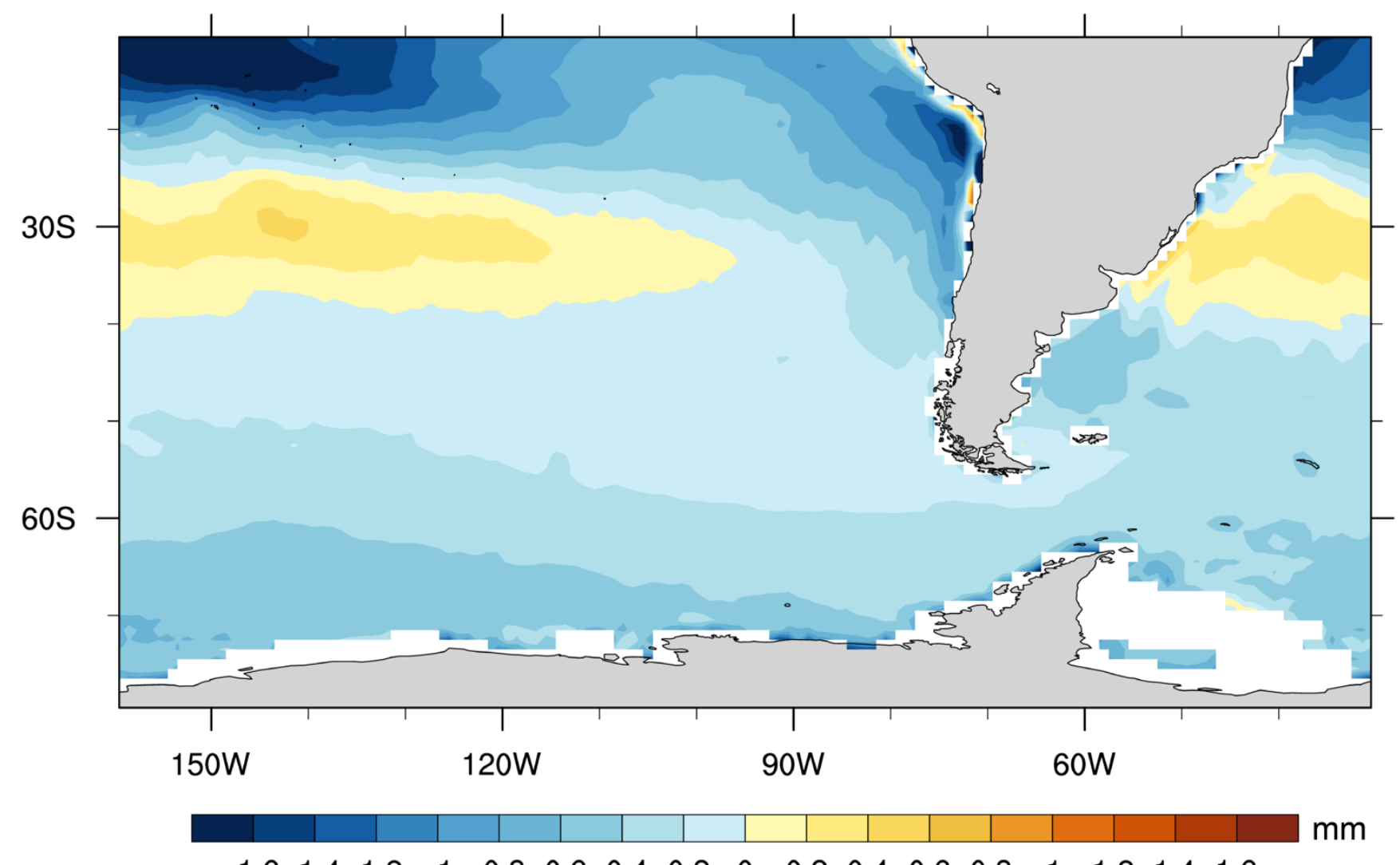

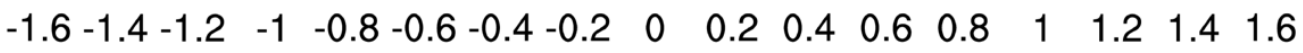

Figure S3: Mean differences in the IWV between ERA Interim and SSMI data for the period 1988-2016. Red shading indicates a positive bias in the ERA Interim data. 


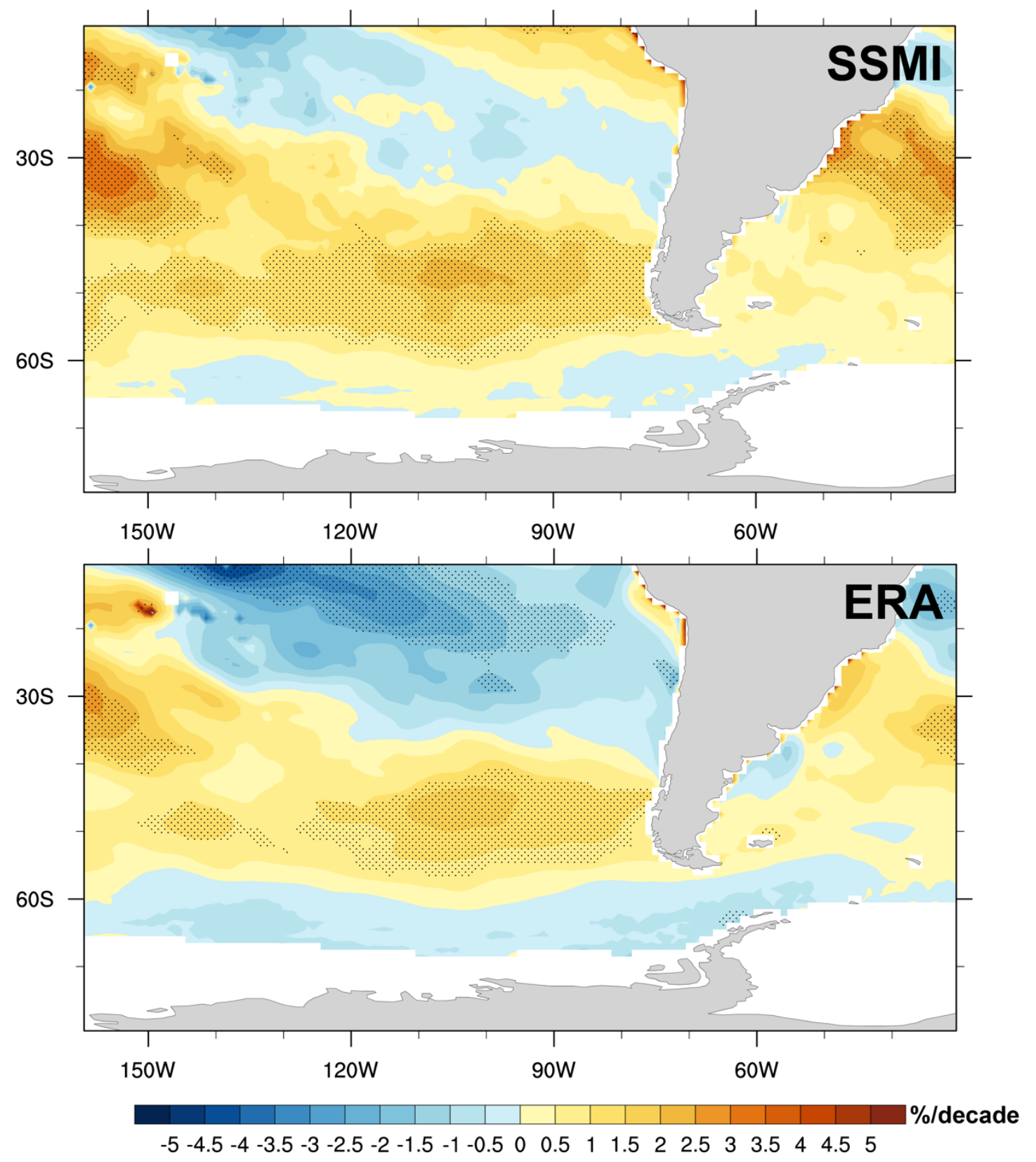

Figure S4: Linear trend of IWV in the SSM/I and ERA-Interim data for the period 1988-2016 (in \% per decade). Dotted areas indicate significant long-term trends $(\mathrm{p}<0.05)$. 


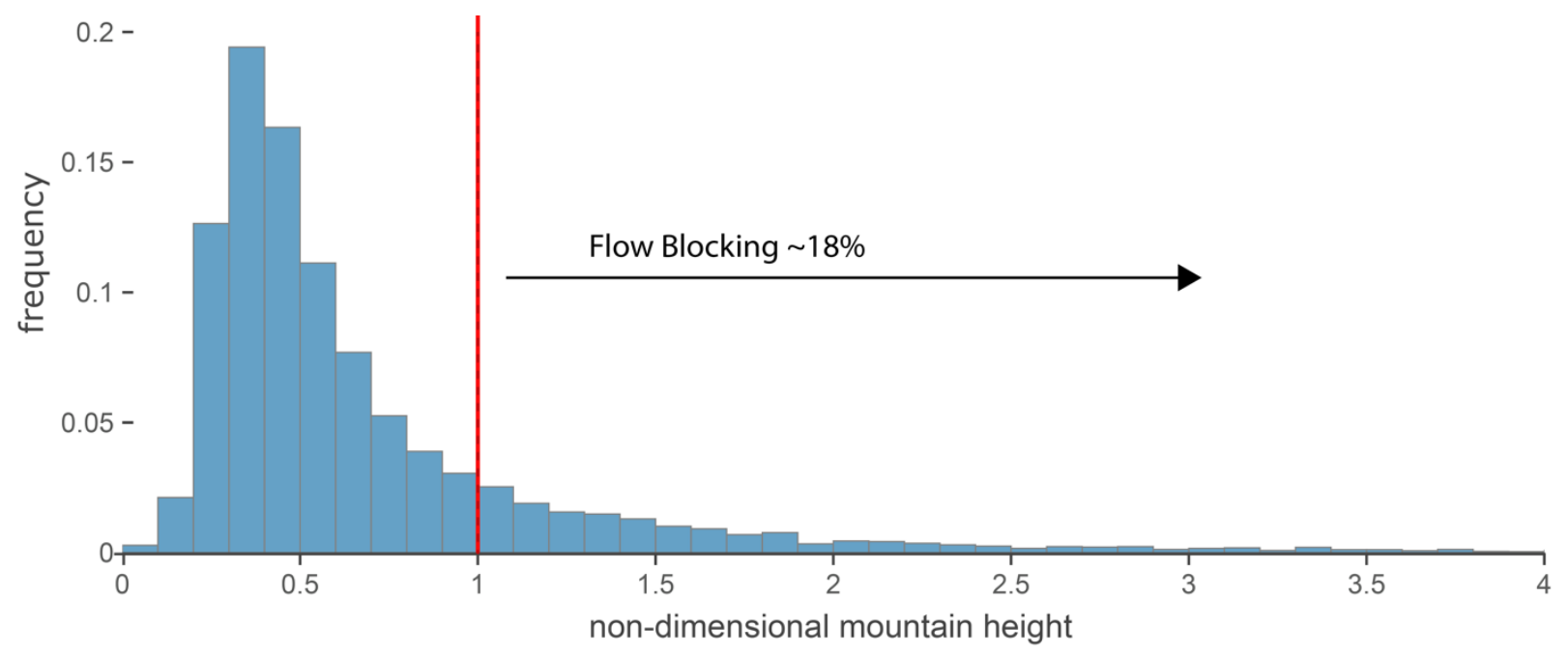

5 Figure S5: Frequency distribution of the non-dimensional mountain height. The non-dimensional mountain height is calculated from ERAInterim data (2010-2016) off Patagonia's west coast (Fig. 1, D1). 
Table S1. Automatic weather stations in Patagonia. Precipitation sums and trends are given in mm. Bold numbers indicate significant trends $(\mathrm{p}<0.05)$.

\begin{tabular}{|c|c|c|c|c|c|c|}
\hline Station & Lat & Lon & Sum & $\begin{array}{c}\text { Trend in } \\
\% \text { per } \\
\text { decade }\end{array}$ & from & to \\
\hline Pirihueico En Pirihueico & -40.02 & -71.72 & 2875 & -0.36 & 1999 & 2015 \\
\hline El Llolly & -40.07 & -72.62 & 1763 & -0.02 & 1995 & 2015 \\
\hline Catamutun & -40.17 & -73.17 & 1858 & 0.02 & 1998 & 2015 \\
\hline Venecia & -40.19 & -73.43 & 3964 & -0.04 & 1998 & 2015 \\
\hline Lago Maihue & -40.22 & -72.15 & 2982 & -0.17 & 1980 & 2015 \\
\hline Trinidad & -40.31 & -73.43 & 1713 & 0.13 & 1998 & 2015 \\
\hline Lago Ranco & -40.32 & -72.47 & 1923 & 0.01 & 1980 & 2015 \\
\hline Adolfo Matthei & -40.59 & -73.11 & 1256 & -0.05 & 1983 & 2016 \\
\hline Canal Bajo Osorno Ad. & -40.61 & -73.06 & 1232 & -0.04 & 1980 & 2016 \\
\hline Anticura & -40.66 & -72.18 & 1185 & -0.39 & 1998 & 2015 \\
\hline Rio Negro En Chahuilco & -40.71 & -73.23 & 1240 & -0.21 & 2004 & 2016 \\
\hline Futacuhuin & -40.72 & -72.44 & 1681 & -0.07 & 1995 & 2015 \\
\hline Rupanco & -40.77 & -72.68 & 1665 & -0.04 & 1994 & 2015 \\
\hline San Antonio Oeste Aero & -40.78 & -65.10 & 202 & 0.25 & 1980 & 2016 \\
\hline Gobernador Castello & -40.87 & -63.00 & 272 & 0.21 & 1980 & 2016 \\
\hline Purranque & -40.94 & -73.14 & 1309 & 0.00 & 1999 & 2015 \\
\hline Frutillar & -41.13 & -73.06 & 1467 & 0.00 & 1994 & 2015 \\
\hline San Carlos De Bariloche & -41.15 & -71.16 & 589 & 0.21 & 1980 & 2016 \\
\hline Fresia & -41.15 & -73.41 & 1613 & -0.03 & 1994 & 2015 \\
\hline La Ensenada & -41.23 & -72.57 & 2358 & 0.00 & 1980 & 2005 \\
\hline Maquinchao & -41.25 & -68.73 & 96 & 0.25 & 1980 & 2016 \\
\hline Lago Chapo & -41.42 & -72.60 & 3038 & -0.05 & 1999 & 2014 \\
\hline El Tepual Puerto Montt Ap. & -41.44 & -73.10 & 1590 & -0.05 & 1980 & 2016 \\
\hline Puerto Montt & -41.46 & -72.94 & 1845 & 0.00 & 1980 & 2016 \\
\hline Puerto Montt & -41.46 & -72.94 & 1845 & 0.00 & 1980 & 2016 \\
\hline Maullin & -41.62 & -73.60 & 1668 & -0.04 & 1987 & 2015 \\
\hline Puelo & -41.65 & -72.31 & 2642 & -0.17 & 1997 & 2016 \\
\hline Ancud 1 (Dga) & -41.86 & -73.82 & 2013 & 0.12 & 1993 & 2015 \\
\hline Hornopiren & -41.94 & -72.44 & 2971 & -0.49 & 1998 & 2016 \\
\hline El Bolson & -41.94 & -71.53 & 232 & 1.02 & 1996 & 2016 \\
\hline
\end{tabular}




\begin{tabular}{|c|c|c|c|c|c|c|}
\hline Chepu & -42.05 & -73.97 & 2554 & -0.08 & 1999 & 2015 \\
\hline Quemchi & -42.14 & -73.47 & 2437 & -0.03 & 2000 & 2015 \\
\hline Castro 1 (Dga) & -42.46 & -73.77 & 1555 & -0.37 & 1993 & 2008 \\
\hline Cucao & -42.62 & -74.11 & 2067 & 0.05 & 1997 & 2015 \\
\hline Chaiten & -42.91 & -72.71 & 3408 & 0.26 & 1998 & 2007 \\
\hline Chaiten Ad. & -42.93 & -72.70 & 3590 & -0.02 & 1980 & 2007 \\
\hline Esquel Aero & -42.93 & -71.15 & 372 & 0.18 & 1980 & 2016 \\
\hline Quellon & -43.11 & -73.61 & 1781 & 0.01 & 1993 & 2015 \\
\hline Puerto. Cardenas & -43.18 & -72.43 & 3922 & -0.33 & 2001 & 2015 \\
\hline Futaleufu Ad. & -43.19 & -71.85 & 1958 & -0.01 & 1980 & 2016 \\
\hline Trelew Aero & -43.20 & -65.27 & 171 & 0.11 & 1980 & 2016 \\
\hline Lago Espolon & -43.22 & -71.93 & 2693 & -0.13 & 2001 & 2015 \\
\hline Valle Rio Frio & -43.47 & -72.35 & 3712 & -0.24 & 2001 & 2015 \\
\hline Christchurch Intl & -43.49 & 172.53 & 486 & 0.05 & 1980 & 2016 \\
\hline Cape Bruny Lighthouse & -43.49 & 147.15 & 940 & -0.07 & 1980 & 2016 \\
\hline Alto Palenaad. & -43.61 & -71.81 & 1589 & -0.04 & 1980 & 2016 \\
\hline Palena & -43.62 & -71.78 & 1597 & -0.20 & 2001 & 2015 \\
\hline Maatsuyker Island Lighthouse & -43.66 & 146.27 & 1235 & -0.16 & 1980 & 2016 \\
\hline Marin Balmaceda & -43.77 & -72.95 & 2388 & 0.17 & 1994 & 2015 \\
\hline Paso De Indios & -43.82 & -68.88 & 86 & 0.44 & 1980 & 1996 \\
\hline Chatham Islands Aws & -43.95 & -176.57 & 624 & 0.16 & 1994 & 2011 \\
\hline La Junta & -43.97 & -72.41 & 1998 & -0.07 & 1981 & 2016 \\
\hline Bordalit & -44.05 & -72.32 & 2508 & -0.29 & 1994 & 2011 \\
\hline Lago Verde & -44.24 & -71.85 & 1244 & 0.41 & 1994 & 2011 \\
\hline Puerto Puyuhuapi & -44.32 & -72.56 & 2994 & -0.08 & 1981 & 2015 \\
\hline Rio Cisnes & -44.50 & -71.31 & 273 & -0.26 & 1981 & 2015 \\
\hline La Tapera & -44.65 & -71.67 & 687 & -0.29 & 1981 & 2002 \\
\hline Cisnes Medio & -44.67 & -72.27 & 2162 & 0.00 & 1982 & 2015 \\
\hline Puerto Cisnes & -44.73 & -72.68 & 2812 & -0.09 & 1981 & 2015 \\
\hline Villa Maihuales & -45.17 & -72.15 & 1399 & -0.09 & 1986 & 2015 \\
\hline Estancia Bao Nuevo & -45.27 & -71.53 & 490 & -0.01 & 2001 & 2015 \\
\hline Irehuao & -45.27 & -71.71 & 409 & 0.11 & 1994 & 2015 \\
\hline Villa Ortega & -45.37 & -71.98 & 654 & -0.14 & 1981 & 2015 \\
\hline Puerto Aysen Ad. & -45.40 & -72.66 & 2011 & -0.11 & 1980 & 2016 \\
\hline Puerto Aysen & -45.40 & -72.70 & 2197 & -0.32 & 1994 & 2008 \\
\hline El Balseo & -45.40 & -72.49 & 1745 & -0.06 & 1981 & 2015 \\
\hline
\end{tabular}




\begin{tabular}{|c|c|c|c|c|c|c|}
\hline Rio Aysen En Puerto Aysen & -45.41 & -72.62 & 1944 & 0.00 & 2003 & 2016 \\
\hline Puerto Chacabuco & -45.46 & -72.82 & 2678 & -0.05 & 1985 & 2015 \\
\hline Coyhaique Alto & -45.48 & -71.60 & 221 & 0.02 & 1985 & 2016 \\
\hline Coyhaique Conaf & -45.55 & -72.06 & 887 & -0.19 & 2003 & 2015 \\
\hline Rio Simpson Bajo Junta Coyhaique & -45.55 & -72.07 & 723 & -0.24 & 2006 & 2016 \\
\hline Coyhaique (Escuela Agricola) & -45.57 & -72.03 & 808 & -0.06 & 1984 & 2016 \\
\hline Teniente Vidal Coyhaique Ad. & -45.59 & -72.11 & 986 & 0.03 & 1980 & 2016 \\
\hline Comodoro Rivadavia & -45.78 & -67.50 & 203 & 0.00 & 1980 & 2016 \\
\hline Balmaceda Ad. & -45.91 & -71.69 & 529 & -0.03 & 1980 & 2016 \\
\hline Villa Cerro Castillo & -46.12 & -72.15 & 540 & -0.41 & 1993 & 2015 \\
\hline Rio Ibaez En Desembocadura & -46.27 & -71.99 & 490 & 0.26 & 2006 & 2016 \\
\hline Puerto Ibaez & -46.29 & -71.93 & 396 & 0.47 & 1986 & 2008 \\
\hline Invercargill Airpor & -46.42 & 168.33 & 888 & 0.01 & 1980 & 2016 \\
\hline Alfred Faure (Iles Crozet) & -46.43 & 51.85 & 1652 & 0.02 & 1980 & 2016 \\
\hline Bahia Murta & -46.46 & -72.67 & 1208 & -0.21 & 1994 & 2015 \\
\hline Perito Moreno Arpt & -46.52 & -71.02 & 74 & -0.08 & 1980 & 2016 \\
\hline Chile Chico & -46.54 & -71.71 & 228 & -0.13 & 1994 & 2015 \\
\hline Chile Chico Ad. & -46.58 & -71.69 & 255 & -0.01 & 1980 & 2016 \\
\hline Puerto Guadal & -46.84 & -72.70 & 565 & 0.31 & 1994 & 2015 \\
\hline Marion Island & -46.88 & 37.87 & 1857 & -0.12 & 1980 & 2016 \\
\hline Estancia Valle Chacabuco & -47.12 & -72.48 & 189 & -0.29 & 1994 & 2015 \\
\hline Rio Baker En Angostura Chacabuco & -47.14 & -72.73 & 745 & 0.05 & 2004 & 2016 \\
\hline Lord Cochrane Ad. & -47.24 & -72.59 & 702 & -0.07 & 1980 & 2016 \\
\hline Rio Cochrane En Cochrane & -47.25 & -72.56 & 480 & 0.19 & 2005 & 2016 \\
\hline Puerto Deseado & -47.74 & -65.90 & 66 & -0.25 & 1980 & 2012 \\
\hline Caleta Tortel & -47.80 & -73.54 & 1987 & -0.13 & 2003 & 2016 \\
\hline Rio Pascua Ante Junta Rio Quetru & -48.16 & -73.09 & 2082 & -0.07 & 2004 & 2016 \\
\hline Rio Mayer Reten & -48.21 & -72.32 & 200 & 1.07 & 1994 & 2003 \\
\hline Villa Ohiggins & -48.47 & -72.56 & 771 & 0.36 & 1994 & 2008 \\
\hline Lago Ohiggins En Villa Ohiggins & -48.52 & -72.60 & 895 & -0.08 & 2004 & 2016 \\
\hline Gobernador Gregores & -48.78 & -70.17 & 63 & -0.85 & 1980 & 1996 \\
\hline Candelario Mancilla & -48.88 & -72.74 & 458 & 0.05 & 1994 & 2016 \\
\hline Puerto Eden & -49.12 & -74.41 & 2346 & -0.43 & 1998 & 2010 \\
\hline San Julian & -49.31 & -67.80 & 190 & 0.09 & 1980 & 2016 \\
\hline Port-Aux-Francais (Iles Kergu & -49.35 & 70.25 & 591 & 0.02 & 1980 & 2016 \\
\hline El Calafate Aero & -50.27 & -72.05 & 145 & -0.16 & 2004 & 2016 \\
\hline
\end{tabular}




\begin{tabular}{|c|c|c|c|c|c|c|}
\hline Lago Argentino Arpt & -50.33 & -72.30 & 120 & 0.98 & 1980 & 1999 \\
\hline Enderby Island Aws & -50.48 & 166.30 & 737 & 0.07 & 1993 & 2016 \\
\hline Lago Dickson & -50.82 & -73.11 & 910 & 0.93 & 2004 & 2016 \\
\hline Cerro Guido & -50.90 & -72.33 & 265 & 0.10 & 1984 & 2016 \\
\hline Rio Las Chinas En Cerro Guido & -51.05 & -72.52 & 226 & 0.29 & 2005 & 2016 \\
\hline Torres Del Paine & -51.18 & -72.97 & 739 & 0.04 & 1983 & 2016 \\
\hline Cerro Castillo & -51.26 & -72.33 & 315 & 0.00 & 1981 & 2016 \\
\hline Rio Gallegos Aero & -51.62 & -69.28 & 214 & 0.19 & 1980 & 2016 \\
\hline Teniente Gallardo Puerto Natales Ad. & -51.67 & -72.53 & 192 & 1.69 & 1999 & 2016 \\
\hline Casas Viejas & -51.70 & -72.33 & 256 & 0.24 & 1981 & 2015 \\
\hline Puerto Natales & -51.73 & -72.48 & 490 & -0.02 & 1986 & 2016 \\
\hline Mount Pleasant & -51.82 & -58.45 & 471 & 0.03 & 1992 & 2016 \\
\hline Rio Rubens En Ruta N 9 & -52.03 & -71.94 & 414 & -1.29 & 2007 & 2016 \\
\hline Teniente Merino & -52.03 & -70.73 & 221 & 0.09 & 1984 & 2015 \\
\hline Rubens En Ruta N. 9 & -52.04 & -71.94 & 473 & -0.52 & 1990 & 2005 \\
\hline Rio Penitente En Morro Chico & -52.05 & -71.42 & 265 & -0.15 & 2007 & 2016 \\
\hline Monte Aymond & -52.16 & -69.61 & 250 & -0.03 & 1996 & 2016 \\
\hline Villa Tehuelche & -52.44 & -71.40 & 337 & -0.04 & 1981 & 2016 \\
\hline Rio Perez & -52.55 & -71.96 & 525 & -0.04 & 1990 & 2014 \\
\hline Campbell Island Aws & -52.55 & 169.17 & 1017 & 0.44 & 1996 & 2016 \\
\hline Seno Skyring & -52.55 & -71.96 & 584 & -0.56 & 2002 & 2016 \\
\hline San Gregorio & -52.57 & -70.07 & 264 & -0.03 & 1992 & 2016 \\
\hline Rio Verde & -52.60 & -71.50 & 337 & 0.02 & 1994 & 2016 \\
\hline Rocallosas & -52.65 & -71.96 & 327 & -0.88 & 1994 & 2015 \\
\hline Cerro Sombrero & -52.78 & -69.29 & 248 & -0.06 & 1984 & 2014 \\
\hline Bahamondes & -52.80 & -72.93 & 3367 & -0.75 & 2000 & 2015 \\
\hline Bahia San Felipe & -52.87 & -69.93 & 351 & 0.12 & 1980 & 2016 \\
\hline Isla Riesco & -52.88 & -71.57 & 408 & -0.09 & 1991 & 2015 \\
\hline Punta Arenas & -53.12 & -70.88 & 923 & 0.02 & 1980 & 2016 \\
\hline Canal De Trasvase Estero Llau-Llau & -53.13 & -70.94 & 648 & -0.10 & 2005 & 2016 \\
\hline Rio Las Minas En Bt. Sendos & -53.14 & -70.99 & 780 & -0.03 & 2000 & 2016 \\
\hline Las Minas & -53.14 & -70.98 & 774 & -0.14 & 1996 & 2015 \\
\hline Laguna Lynch & -53.14 & -70.98 & 435 & -0.04 & 1980 & 2015 \\
\hline Fuentes Martinez Porvenir Ad. & -53.19 & -70.32 & 260 & -0.03 & 1986 & 2016 \\
\hline Porvenir & -53.29 & -70.37 & 315 & -0.20 & 1991 & 2015 \\
\hline Onaisin En Maria Cristina & -53.31 & -69.27 & 315 & -0.12 & 1990 & 2016 \\
\hline
\end{tabular}




\begin{tabular}{lrrrrrr} 
San Sebastian & -53.32 & -68.66 & 294 & 0.13 & 1990 & 2016 \\
Lago Parrillar & -53.40 & -71.25 & 800 & 0.00 & 1990 & 2016 \\
Cameron & -53.64 & -69.65 & 366 & 0.08 & 1994 & 2016 \\
San Juan & -53.65 & -70.96 & 544 & $\mathbf{0 . 2 9}$ & 1980 & 2016 \\
Russfin & -53.76 & -69.19 & 429 & -0.01 & 1994 & 2016 \\
Rio Caleta En Tierra Del Fuego & -53.86 & -70.00 & 340 & -0.15 & 2007 & 2016 \\
Rio Grande En Tierra Del Fuego & -53.89 & -68.88 & 276 & 0.14 & 2007 & 2016 \\
Seccion Rio Grande & -53.90 & -68.92 & 401 & 0.15 & 1991 & 2011 \\
Pampa Huanaco & -54.05 & -68.80 & 340 & $\mathbf{0 . 2 8}$ & 1994 & 2016 \\
Macquarie Island & -54.50 & 158.94 & 912 & -0.03 & 1980 & 2016 \\
Ushuaia Malvinas Argentinas & -54.84 & -68.30 & 335 & 0.24 & 1980 & 2016 \\
Guardia Marina Zanartu Pto Williams & -54.93 & -67.62 & 466 & -0.09 & 1980 & 2016 \\
Rio Robalo En Puerto Williams & -54.95 & -67.64 & 389 & 0.14 & 2005 & 2016 \\
\hline
\end{tabular}

Table S2. Comparison of the IWV trends between atmospheric soundings and the nearest ERA-Interim grid point. Numbers are given in $5 \mathrm{~mm}$ decade ${ }^{-1}$. Bold numbers indicate significant trends $(\mathrm{p}<0.05)$.

\begin{tabular}{llll}
\hline & $\mathbf{1 9 8 8 - 2 0 1 6}$ & $\mathbf{1 9 8 8 - 2 0 0 9}$ & $\mathbf{2 0 1 0 - 2 0 1 6}$ \\
\hline Puerto Montt & & & \\
Radiosounding & $-0.22(-1.5 \%)$ & $-0.42(-3.0 \%)$ & $2.87(19.4 \%)$ \\
ERA-Interim & $-0.16(-1.3 \%)$ & $-0.25(-2.0 \%)$ & $0.65(5.2 \%)$ \\
& & & \\
Punta Arenas & & & $1.23(11.3 \%)$ \\
Radiosounding & $0.23(2.2 \%)$ & $0.07(0.6 \%)$ & $0.80(7.0 \%)$ \\
ERA-Interim & $0.14(1.3 \%)$ & $0.05(0.4 \%)$ & \\
\hline
\end{tabular}

Table S3. Comparison of the $\mathrm{OPM}_{0.45}$ and $\mathrm{OPM}_{0.60}$ experiments with observations. Given are the latitude (lat), longitude (lon), altitude (alt), the precipitation values from the orographic precipitation model experiments $\left(\mathrm{OPM}_{0.45}\right.$ and $\left.\mathrm{OPM}_{0.60}\right)$ and the observations $(\mathrm{Obs})$. The

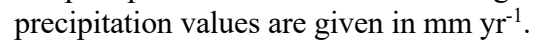

\begin{tabular}{lcccccc}
\hline Location & lat & lon & alt & OPM $_{\mathbf{0 . 4 5}}$ & OPM $_{\mathbf{0 . 6 0}}$ & Obs \\
\hline Villa Cerro Castillo & -46.12 & -72.15 & 345 & 471 & 693 & 282 \\
Rio Ibaez En Desembocadura & -46.26 & -71.99 & 220 & 298 & 463 & 623 \\
Bahia Murta & -46.46 & -72.66 & 240 & 849 & 1275 & 1017
\end{tabular}




\begin{tabular}{|c|c|c|c|c|c|c|}
\hline Lago General Carrera Fachinal & -46.54 & -72.22 & 18 & 579 & 823 & 333 \\
\hline Glaciar San Rafael & -46.64 & -73.85 & 8 & 3829 & 5198 & 1271 \\
\hline Puerto Guadal & -46.84 & -72.70 & 210 & 745 & 1141 & 656 \\
\hline Estancia Valle Chacabuco & -47.11 & -72.48 & 343 & 613 & 874 & 159 \\
\hline Rio Nef Antes Junta Estero El Revalse & -47.13 & -73.08 & 281 & 895 & 1405 & 974 \\
\hline Rio Baker En Angostura Chacabuco & -47.14 & -72.72 & 160 & 673 & 1029 & 856 \\
\hline Lago Cachet 2 En Glaciar Colonia & -47.19 & -73.25 & 427 & 1088 & 1676 & 243 \\
\hline Lord Cochrane Ad. & -47.24 & -72.58 & 204 & 744 & 1056 & 652 \\
\hline Rio Cochrane En Cochrane & -47.25 & -72.56 & 140 & 788 & 1094 & 514 \\
\hline Rio Colonia En Nacimiento & -47.33 & -73.11 & 146 & 986 & 1526 & 1261 \\
\hline Caleta Tortel & -47.79 & -73.53 & 10 & 2389 & 3318 & 1870 \\
\hline Rio Pascua Ante Junta Rio Quetru & -48.15 & -73.08 & 20 & 1668 & 2342 & 2137 \\
\hline Lago Ohiggins En Villa Ohiggins & -48.51 & -72.59 & 300 & 752 & 1084 & 909 \\
\hline Candelario Mancilla & -48.87 & -72.73 & 300 & 850 & 1276 & 519 \\
\hline Rio Punta Eva En Puerto Eden & -49.11 & -74.41 & 10 & 3607 & 5429 & 2840 \\
\hline El Calafate Aero & -50.26 & -72.05 & 204 & 433 & 586 & 149 \\
\hline Lago Dickson & -50.82 & -73.11 & 200 & 1301 & 1906 & 1130 \\
\hline Lago Paine & -50.84 & -72.90 & 440 & 1132 & 1671 & 500 \\
\hline Cerro Guido & -50.89 & -72.33 & 230 & 820 & 1097 & 312 \\
\hline Amalia & -50.95 & -73.69 & 0 & 4350 & 5539 & 2801 \\
\hline Rio Paine En Parque Nacional 2 & -50.96 & -72.79 & 90 & 1139 & 1675 & 724 \\
\hline Nunatak Grey & -50.97 & -73.22 & 300 & 1414 & 2059 & 589 \\
\hline Lago Sarmiento & -51.01 & -72.71 & 110 & 1111 & 1633 & 352 \\
\hline Lago Pehoe & -51.07 & -72.99 & 40 & 1347 & 1966 & 868 \\
\hline Lago Grey & -51.11 & -73.13 & 50 & 1397 & 2064 & 663 \\
\hline Glaciar Tindall & -51.11 & -73.28 & 345 & 1584 & 2282 & 1200 \\
\hline Torres Del Paine & -51.18 & -72.96 & 25 & 1431 & 2106 & 750 \\
\hline Rio Rincon En Ruta Y-290 & -51.31 & -72.82 & 36 & 1554 & 2285 & 769 \\
\hline Rio Serrano En Desembocadura & -51.33 & -73.10 & 25 & 1671 & 2456 & 1227 \\
\hline
\end{tabular}

Table S4. Summary of the WRF configuration. 


\section{Domain configuration}

Horizontal grid spacing

$12.5-\mathrm{km}, 2.5-\mathrm{km}$, and $500-\mathrm{m}$

Vertical levels 55

Model top pressure

$100 \mathrm{hPa}$

\section{Model physics}

Radiation

RRTMG

Microphysics

Morrison

Cumulus

Kain-Fritsch

Planetary boundary layer

MYNN Level 2.5

Atmospheric surface layer

Monin Obukhov

Land surface

Noah-MP

Top boundary condition

Rayleigh damping

\section{Lateral boundaries}

Forcing

ERA-Interim

$0.75^{\circ} \times 0.75^{\circ}, 6$-hourly 